\title{
On the emerging role of body culture and individualised exercise training for PPPM
}

\author{
Henning Boecker
}

Published online: 28 September 2011

(C) European Association for Predictive, Preventive and Personalised Medicine 2011

"Mens sana in corpore sano" ("It is to be prayed that the mind be sound in a sound body"; http://en.wikipedia.org/ wiki/Mens_sana_in_corpore_sano). This famous quotation from Roman poet Juvenal (late 1st and early 2 nd century $\mathrm{AD}$, author of the "Satires") seems more than ever up-todate as, indeed, common beliefs are now increasingly backed up by empirical and experimental scientific evidence. The role of regular moderate physical exercise for both, bodily and mental health, is of fundamental importance when facing the massive health burden promoted by increasing sedentary lifestyles and changing alimentation habits, not only in western civilisations. Exercise is associated with positive health outcomes, including reduction of cardiovascular risk factors (hypertension, hypercholesterolemia, obesity, etc.) and associated cardiovascular mortality, and there is emerging evidence to support preservation of central nervous system functioning with positive health outcomes to depression and anxiety, sleep pain regulation and, most notably, in cognitive domains like memory and attention.

"PPPM in Body Culture and Individualised Exercise Training" has been launched in the understanding, that based on solid evidence from basic research, clinical research, and clinical studies - recommendations for personalised preventative strategies can be launched within the framework of the EPMA society. The umbrella term "Body Culture and Individualised Exercise Training" covers a wide spectrum of topics, only some very few of

H. Boecker $(\bowtie)$

Radiologische Universitätsklinik,

FE Klinische Funktionelle Neurobildgebung,

Rheinische Friedrich-Wilhelms-Universität Bonn,

Sigmund-Freud-Str. 25,

53127 Bonn, Germany

e-mail: Henning.Boecker@ukb.uni-bonn.de which are represented in this first dedicated issue of the EPMA journal; notably, it is intended for the future to host articles related to personalised prevention strategies, but also more work focusing on biomarkers that can be applied for monitoring of physical interventions, possibly on the individual level, but also for individually tailored exercise regimens.

In this first issue, Pekka Oja and Sylvia Titze from Tampere, Finland, nicely review the current state of physical activity recommendations for public health [1], with a particular focus on Europe. While most European countries have integrated physical activity recommendations in their national health policies, the authors make the important point that there is a need for continued update, based on emerging scientific evidence. While population-targeted physical activity recommendations have been conceptualised to inform the general public as well as health policy makers on "what kind and how much physical activity is needed for health benefits", they are "not meant to be used as such for individualised exercise prescriptions for the treatment of specific diseases". This will be an important path to be pursued in future.

The prevalence of juvenile obesity is increasing worldwide and is associated with elevated risk of cardiovascular and metabolic disease, but also considerable psychological and social consequences. Christine Graf, a distinguished expert from the Deutsche Sporthochschule in Cologne, contributes to this important PPPM issue with a highly informative and well structured review article on current state-of-the-art knowledge regarding prevention and treatment of juvenile obesity [2], but also covering issues like "prenatal programming" of obesity, the critical role of visceral fat, etc. Her review makes the point that measures should start as early as possible by integrating the family / environment of affected children and adolescents, and by launching educational strategies. 
Henning Boecker, Bonn, reviews the current role of neuroimaging for monitoring of exercise-related effects on brain structure and function [3]. His paper claims an "emerging role of neuroimaging in determining functional and structural brain integrity induced by physical exercise" and makes the point, that modern neuroimaging approaches, in particular magnetic resonance imaging (MRI) based techniques provide a powerful tool for studying the effects of physical exercise on human brain structure and function non-invasively. Despite its potential to uncover central mechanisms of exercise on a between-group basis, or, even more interestingly, on a within-group longitudinal basis in healthy subjects, at-risk cohorts, and patient populations, the review is critical regarding its current applicability as a biomarker in individual subjects. Rather, it is expected that neuroimaging findings from larger cohorts "may propagate individualized exercise training regimens for disease prevention and, ultimately, adapted applications for prevention and slowing down disease progression in neurodegenerative conditions".

Dimiter V. Dimitrov et al. from Varna, Bulgaria, shed light on the current indications and the post-operative follow-up of patients undergoing bariatric surgery for treatment of obesity [4]. Bariatric medicine refers to gastrointestinal surgery conducted to produce sustainable weight loss and includes laparoscopic techniques like "gastric banding, gastric bypass, sleeve gastrectomy, biliopancreatic diversion", and more recently, implantable gastric stimulation. In certain, highly selected patient groups that do not achieve recommended treatment targets with medical therapies, these invasive techniques are currently considered on an individual basis, where the risk associated with the invasive intervention has to be weighted against long-term consequences of unfavourable cardiovascular risk profiles. The choice of procedures is based on knowledge of the results, long-term effects, complications, and individual circumstances. In any case, subsequent multidisciplinary and lifelong multidisciplinary follow-up after bariatric surgery is mandatory, to prevent metabolic deficiencies and because there is an increased risk for weight regain.

There is evidence that exercise has effects on mood, and may propagate antidepressive effects by acting on serotonergic and opiodergic / dopaminergic neurotransmission. This is an interesting topic for future issues of "PPPM in Body Culture and Individualised Exercise Training". It is important, therefore, that three further articles of this issue set the path by highlighting the relationship between insomnia and depression [5] (Chiara Baglioni et al.; Freiburg, Germany), sleep disturbances and suicidality [6] (Christine Norra et al.; Bochum, Germany) and treatment effects of cognitive behavioural therapy on insomnia [7] (Tatjana Crönlein and Jürgen Zulley; Regensburg, Germany).

\section{References}

1. Oja P, Titze S. Physical activity recommendations for public health: development and policy context. EPMA J. 2011. doi:10.1007/ s13167-011-0090-1.

2. Graf C. Preventing and treating obesity in pediatrics through physical activity. EPMA J. 2011. doi:10.1007/s13167-011-0091-0.

3. Boecker $\mathrm{H}$. On the emerging role of neuroimaging in determining functional and structural brain integrity induced by physical exercise: impact for predictive, preventive, and personalized medicine. EPMA J. 2011. doi:10.1007/s13167-011-0093-y.

4. Dimitrov DV, Ivanov V, Atanasova M. Advantages of bariatric medicine for individualized prevention and treatments: multidisciplinary approach in body culture and prevention of obesity and diabetes. EPMA J. 2011. doi:10.1007/s13167-011-0099-5.

5. Baglioni C, Spiegelhalder K, Nissen C, Riemann D. Clinical implications of the causal relationship between insomnia and depression: how individually tailored treatment of sleeping difficulties could prevent the onset of depression. EPMA J. 2011. doi:10.1007/s13167-011-0079-9.

6. Norra C, Richter N, Juckel G. Sleep disturbances and suicidality: a common association to look for in clinical practise and preventive care. EPMA J. 2011. doi:10.1007/s13167-011-0101-2.

7. Crönlein T, Zulley J. The options available in cognitive behavioral therapy to prevent chronification of insomnia. EPMA J. 2011. doi:10.1007/s13167-011-0095-9.

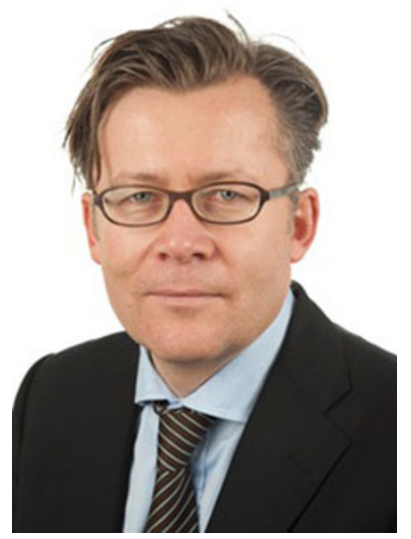

Henning Boecker has received his medical degree and doctorate from the University of Düsseldorf Medical School, and went on to specialise in neurology and nuclear medicine at the Technical University in Munich. He received his lecturer's qualification in 2002 was appointed in 2005 as Full Professor and Head of the Division Functional Brain Imaging at the Department of Radiology (Prof. Dr. H.H. Schild), University of Bonn. His methodological spectrum covers various imaging modalities for in vivo structural and functional neuroimaging, including positron emission tomography (PET) and magnetic resonance imaging (MRI). His research interests cover neurodegenerative disorders, in particular movement disorders, and, more recently, using multimodal neuroimaging to study the effects of exercise on brain structure and function. He serves as editorial board member of several scientific journals such as The EPMA Journal, the Open Access Journal of Sports Medicine, and the American Journal of Nuclear Medicine and Molecular Imaging. 